\title{
Spotlight on dupilumab in the treatment of atopic dermatitis: design, development, and potential place in therapy
}

\author{
This article was published in the following Dove Press journal: \\ Drug Design, Development and Therapy \\ 15 May 2017 \\ Number of times this article has been viewed
}

\author{
Angelo Massimiliano \\ D’Erme ${ }^{1,2}$ \\ Marco Romanelli ${ }^{2}$ \\ Andrea Chiricozzi ${ }^{2}$ \\ 'Dermatology Unit, Livorno \\ Hospital, Livorno, ' $D$ Dermatology \\ Unit, Department of Clinical and \\ Experimental Medicine, University \\ of Pisa, Pisa, Italy
}

\begin{abstract}
Atopic dermatitis (AD) is among the most common inflammatory skin diseases in children and adults in industrialized countries. Up to one-third of adults (probably a smaller proportion in childhood) suffer from moderate-to-severe AD, whose recommended treatment is usually based on systemic therapies. The currently available therapeutics are limited, and $\mathrm{AD}$ management becomes challenging in most cases. Over the last few years, new advances in the understanding of $\mathrm{AD}$ pathogenic mechanisms and inflammatory pathways have led to the identification of specific therapeutic targets and new molecules have been tested. Dupilumab is a fully human monoclonal antibody directed against the IL-4 receptor $\alpha$ subunit that is able to block the signaling of both IL-4 and IL-13 and achieve rapid and significant improvements in adults with moderate-to-severe AD. Dupilumab is ready to inaugurate a long and promising biological target treatment option for Th2 cell-mediated atopic immune response that characterizes AD.
\end{abstract}

Keywords: dupilumab, atopic dermatitis, eczema, IL-4, IL-13, biologics

\section{Introduction}

Atopic dermatitis (AD) is one of the most common inflammatory skin diseases affecting up to $25 \%$ of children in industrialized countries. ${ }^{1}$ One-third of cases persist into adulthood, comprising a prevalence of $2 \%-10 \% .^{2-4} \mathrm{AD}$ is not just a skin disease as it may represent the first manifestation of the so-called atopic march, a spectrum of interconnected disorders, including rhinitis, conjunctivitis, and asthma, that may follow skin symptoms later in life. ${ }^{2-6}$

Because the prevalence of AD is lower in rural and nonindustrialized countries, ${ }^{4}$ the hygiene hypothesis, in which the lack of exposure to antigens in early life would induce immune imbalance, favoring a proinflammatory Th2 response that drives the immune dysregulation in $\mathrm{AD}$, has been proposed. ${ }^{4,7,8}$

\section{Immunopathogenesis of AD}

AD pathogenesis represents a complex mechanism, including a defective epidermal barrier, caused by an altered expression of keratinocyte differentiation genes (eg, cornified cell envelope-related genes) and an abnormal content of extracellular lipids, resulting in increased transepidermal water loss and permeation to allergens, irritants, and microbes. ${ }^{9-11}$

Beside this intrinsic impairment of the keratinocyte differentiation process, $\mathrm{AD}$ lesional skin shows a marked infiltration of $\mathrm{T}$ cells, both $\mathrm{CD} 4^{+}$and $\mathrm{CD}^{+}$

\footnotetext{
Correspondence: Angelo Massimiliano D'Erme

Dermatology Unit, Livorno Hospital,

Viale Alfieri, Livorno 57124, Italy

Tel +39349845 I303

Email a.m.derme@gmail.com
} cc) ${ }_{\mathrm{BY}} \mathrm{NC}$ and incorporate the Creative Commons Attribution - Non Commercial (unported, v3.0) License (http://creativecommons.org/licenses/by-nc/3.0/). By accessing the work you hereby accept the Terms. Non-commercial uses of the work are permitted without any further permission from Dove Medical Press Limited, provided the work is properly attributed. For permission for commercial use of this work, please see paragraphs 4.2 and 5 of our Terms (https://www.dovepress.com/terms.php). 
T cells, dendritic cells (DCs), Langerhans cells, and other immune cells, including eosinophils, mast cells, and IgEproducing plasma cells. ${ }^{9}$ Classically, AD is considered as a Th2-dominant disease, as an enhanced signal of the Th2 pathway is detected in AD lesional skin and, to a lesser strength, in non-lesional skin. ${ }^{5,12,13}$ The increased expression of Th2-derived cytokines, namely, IL-4, IL-5, IL-13, and IL-31, is correlated with high levels of Th2 chemoattractants and activating factors such as CCL-17, CCL-18, CCL-22, CCL-26, CCL-27, and TSLP. ${ }^{14,15}$

\section{IL-4 and IL- 13}

The centrality of the Th2 cytokines is due to their capability of 1) inducing IgE class switching; 2) promoting Th2 survival; 3) recruitment of eosinophils; 4) mediating pruritus; and 5) inhibiting keratinocyte terminal differentiation and AMP production (Figure 1). Particularly, IL-4 and IL-13 are considered as the Th2-signature cytokines and master mediators in AD pathogenesis as they act on various cells involved in AD (ie, keratinocytes, T cells, DCs, and eosinophils), signaling through the same receptor, the IL- $4 \mathrm{R} \alpha$ receptor. Although the immune response is polarized toward a Th2 response, other T-cell subsets participate in AD pathogenesis, including T22 cells and both $\mathrm{CD}^{+}$and $\mathrm{CD}^{+} \mathrm{T}$ cells producing IL-22-, IL-17-, and IFN $\gamma$-secreting cells. ${ }^{16-19}$ Based on the dominant pathways driving $\mathrm{AD}$ inflammation, intrinsic $\mathrm{AD}$ may be distinguished from the extrinsic form. Indeed, the extrinsic form ( $~ 80 \%$ of AD cases) shows high IgE serum levels associated with a Th2-skewed immune polarization and a less pronounced T22 signal, whereas the intrinsic form (the remaining 20\%) is characterized by low IgE titers and a Th2 response, with a marked upregulation of the Th17 and Th22 axes. ${ }^{20}$ In this scenario, key mediators, such as IL-4, IL-13, and IL-22, have been identified as therapeutic targets

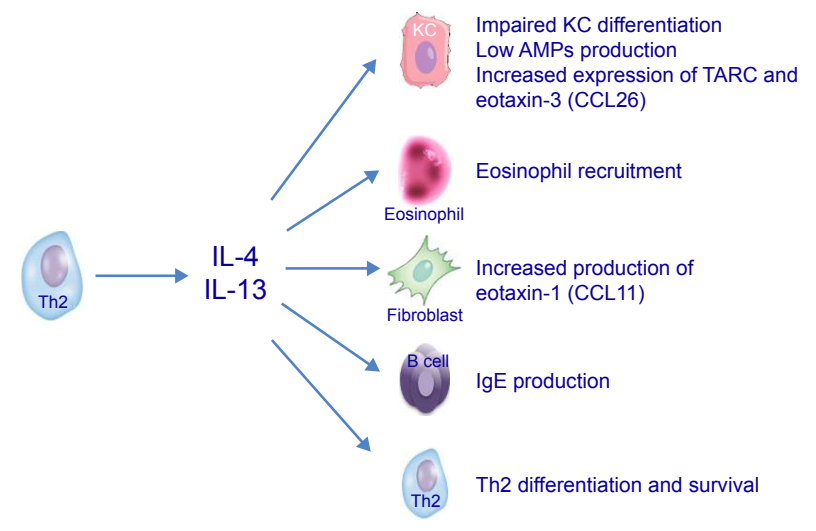

Figure I Th2-derived cytokines whose activity is neutralized by dupilumab antagonizing their receptor. for the development of new agents that selectively inhibit their signaling. One of the promising agents that is currently being developed for the treatment of $\mathrm{AD}$ is dupilumab, an IL-4R $\alpha$ antagonist.

\section{Clinical phenotypes and endophenotypes of AD toward personalized treatment}

$\mathrm{AD}$ is characterized by a wide range of heterogeneity either in the onset (ie, infant-adolescent-adult), course, and presentation (different manifestation of eczema among either the age, the clinical features, or the area involved) or in the comorbidities (eg, the presence of atopy and normal IgE distinguishes an intrinsic AD from an extrinsic or IgE-associated AD). ${ }^{21,22}$ All these variants are due to the complex interactions between individual genetic and environmental factors involved in $\mathrm{AD}$ that lead to epidermal barrier dysfunction, innate and adaptive abnormalities of the immune system (an initial Th2 phase followed by a chronic Th1 phase), and cutaneous microbiome dysbiosis. Despite all these AD variants, the diagnosis is clinical and no diagnostic biomarkers dissecting AD from other inflammatory disorders have been identified yet. ${ }^{23-27}$ Since $\mathrm{AD}$ involves more than one subtype, the discovery, validation, and use of objective markers will achieve a more personalized clinical and treatment approach. ${ }^{28}$

Nowadays, treatment choice is based on disease severity and symptoms, namely, pruritus and the "trouble sleeping". The commonly used assessment tools, both in trial setting and in daily practice, include the Eczema Area and Severity Index (EASI), a composite score assessing objective signs, and the SCORing Atopic Dermatitis (SCORAD), evaluating both signs and symptoms. Management and treatment in the short (flares) and long periods may become challenging because of the individual symptomatic variability and the limited array of therapeutics currently available. ${ }^{29-31}$

\section{Current treatment for AD}

AD treatment includes "basic therapy" focused on emollients and moisturizing compounds, on the avoidance of specific or unspecific triggering factors, and on educational programs/ psychological counseling. ${ }^{30-32}$

First-line pharmacological treatment is based on the use of topical medications: topical corticosteroids and topical calcineurin inhibitors, namely, tacrolimus and pimecrolimus. Topical agents represent the mainstay of therapy in patients with mild-to-moderate disease and are used either for the management of exacerbation or, more recently, for proactive therapy. ${ }^{29-31,33}$ The long-term intermittent (twice weekly) 
anti-inflammatory therapy aims to prevent relapses and to keep the skin free of eczema. This strategy has been proven successful in improving the clinical management and is, notably, associated with pharmacoeconomic benefits. ${ }^{29-31}$

Phototherapy (ultraviolet A1 [UVA1] wavelength or ultraviolet B [UVB] $311 \mathrm{~nm}$ ) can be adjuncted in controlling challenging cases not responsive to topical treatments, such as second-line treatment, with good results. ${ }^{29-31}$

Up to one-third of adults (probably a smaller proportion in childhood) suffer from moderate-to-severe $\mathrm{AD}$, whose recommended treatment is usually based on systemic therapies. The treatment choice depends on AD severity, patient's features, physician's experience, and drug availability. ${ }^{5}$ Although the majority of evidence exists in adult populations, four systemic immunosuppressive drugs have also demonstrated to be efficacious in adults and children: cyclosporine, mycophenolate mofetil, methotrexate, and azathioprine. ${ }^{33,34}$ Among them, cyclosporine is the only drug approved for the treatment of AD. According to the European Guidelines, other systemic medications are suggested in case of no response to cyclosporine and/or when contraindications to cyclosporine occur. ${ }^{29-31,33}$ No biologic drugs targeting ADsignature mediators have been approved yet. Nevertheless, off-label use, sometimes successful, of biologics approved for other indications, such as omalizumab, ustekinumab, and rituximab, has been reported. ${ }^{35}$

Thus, the current therapeutic paradigm in AD is limited and its management becomes challenging in most cases. Furthermore, some side effects of these medications, such as nephrotoxicity and risk of hypertension during cyclosporine treatment, limit their use.

\section{Dupilumab: developing the new era in the treatment of AD}

Over the last few years, new advances in the understanding of AD pathogenic mechanisms and inflammatory pathways have led to the identification of specific therapeutic targets, similar to what occurred in the past decade in psoriasis. ${ }^{28,36}$ Th2 cells and their key cytokines, such as IL-4, IL-13, IL-5, TSLP, and IgE, constitute emerging targets for new compounds that have been or are currently being tested in clinical trials. ${ }^{28,37}$ In the last few years, some biologics, such as omalizumab (anti-IgE) and mepolizumab (antiIL-5), have been previously tested in patients with AD, although no significant benefits were described. ${ }^{33,38-40} \mathrm{On}$ the contrary, the blockade of IL-4/IL-13-mediated signaling through the receptor antagonism was proven to be a successful therapeutic strategy in AD. Dupilumab is a fully human monoclonal antibody directed against the IL-4 receptor $\alpha$ subunit, which is a component of Type I and Type II IL-4 receptors and the IL-13 receptor system. Because of this binding, dupilumab is able to effectively inhibit the IL-4 and IL-13 signaling, with pivotal effect in Th2 inflammatory atopic response (Figure 1). ${ }^{41}$ IL-4 and IL-13 increase the expression of important chemokines such as TARC and eotaxin-3 and attract Th2 cells and eosinophils. They are also important in $\mathrm{AD}$ because they contribute directly to barrier dysfunction (acting on keratinocyte differentiation and barrier protein, lipids, and production of antimicrobial peptides). ${ }^{42}$

\section{Mechanistic study}

To define dupilumab's spectrum of action on AD skin, a comprehensive gene expression analysis was performed, comparing pretreatment with posttreatment lesional skin and non-lesional skin. Biopsy specimens were obtained from 18 adult patients with moderate-to-severe chronic AD participating in two Phase I multicenter, randomized, doubleblind, placebo-controlled trials testing weekly subcutaneous injections of dupilumab 150 or $300 \mathrm{mg}$ or placebo for 4 weeks. ${ }^{42}$ Disease severity, assessed by EASI, was significantly improved in adults treated with dupilumab compared to the placebo group as EASI-50, representing at least 50\% reduction in the EASI score relative to baseline, was achieved by all but one patient in the substudy treated with $300 \mathrm{mg}$ of dupilumab versus none in the placebo group. ${ }^{42}$

Clinical improvement was associated with significant dose-dependent changes from baseline in the defined AD transcriptome (meant as the array of differentially expressed genes between lesional and non-lesional AD skin) detected in lesional skin by using microarrays in the dupilumab group compared with the placebo arm of the study. ${ }^{13}$ The altered expression detected in pretreatment lesional skin improved after dupilumab treatment, with 821 genes whose expression was modulated by $300 \mathrm{mg}$ dupilumab versus 275 placebomodulated probes. ${ }^{42}$ In particular, dupilumab downregulated a large set of genes codifying for inflammatory mediators and epidermal proliferation markers, while it upregulated genes involved in the structural, lipid metabolism, and barrier-related functions. Particularly, dupilumab was able to suppress key pathogenic circuits mediated by Th2-derived products, as demonstrated by the reduction in IL-4 and IL-13 levels at skin lesions observed in dupilumab-responder patients. ${ }^{42}$ As confirmed by quantitative real-time reverse transcription polymerase chain reaction (qRT-PCR), dupilumab was able to suppress the expression of proinflammatory genes related 
not only to a Th2 and eosinophil response (eg, CCL13 and CCL26) but also to the IL-17/IL-22 signaling and Th1 pathway. ${ }^{42}$ Overall, these mechanistic changes induced by dupilumab, including the suppression of immune cell activation (ie, T cells, DCs, and eosinophils) and the amelioration of keratinocyte proliferation and differentiation, were correlated with clinical improvement of $\mathrm{AD}$, and these effects were dose dependent in dupilumab-treated patients.

\section{Phase I and II studies}

The first controlled studies (Phase I and II) testing dupilumab for the treatment of AD were performed in 2014. ${ }^{35,43}$ In adults with moderate-to-severe $\mathrm{AD}$, dupilumab, administered as 4- to 12-week monotherapy or in association with topical corticosteroids, achieved rapid and significant improvements.

Reduction in disease severity was observed, starting from the first week, obtaining 74\% decrease in EASI after 12 weeks of treatment. Similarly, a significant reduction in pruritus levels $(55.7 \%$ in the treatment group versus $15 \%$ in the placebo group) was noted, with positive repercussion on patients' quality of life (Table 1). Clinical improvements were proportional to the drug dose. ${ }^{43,44}$ During the dupilumab treatment, early and sustained improvement of patientreported outcomes (PROs) in sleep, mental health, and health-related quality of life were highlighted. ${ }^{43,45}$

Safety data showed no evidence of drug-related serious adverse events or organ toxicity, with nasopharyngitis and headache among the most common side effects. ${ }^{44}$

The clinical improvement was correlated with Th2-related biomarker changes at serum and tissue levels and normalization of gene expression in lesional skin, appearing similar to the non-lesional gene expression profile. ${ }^{43}$ The latter data suggest that the epidermal abnormalities associated with AD might be somehow reduced with dupilumab treatment. ${ }^{43,46}$

Overall, the vigorous effects of dupilumab in improving clinical and laboratory signs confirm the pivotal pathogenic role of IL-4 and IL-13 signaling in adulthood AD and further support the use of Th2 cytokine antagonists in the treatment of this disease..$^{43,47}$

\section{Phase III trials}

In 2016, two randomized, placebo-controlled, worldwide Phase III trials were conducted, enrolling 671 adult patients suffering from moderate-to-severe AD whose disease was inadequately controlled by topical treatment (patients suffering from $\mathrm{AD}$ variants were included, eg, intrinsic, extrinsic, with early onset, or with late onset). In these studies, dupilumab significantly improved the signs and symptoms

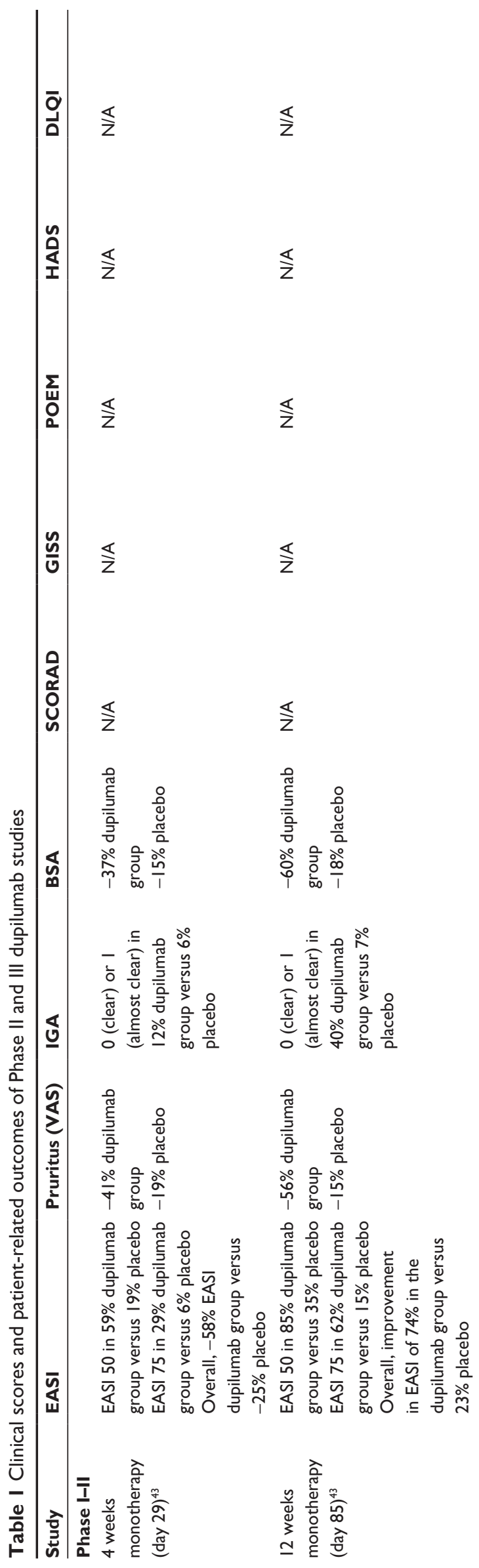




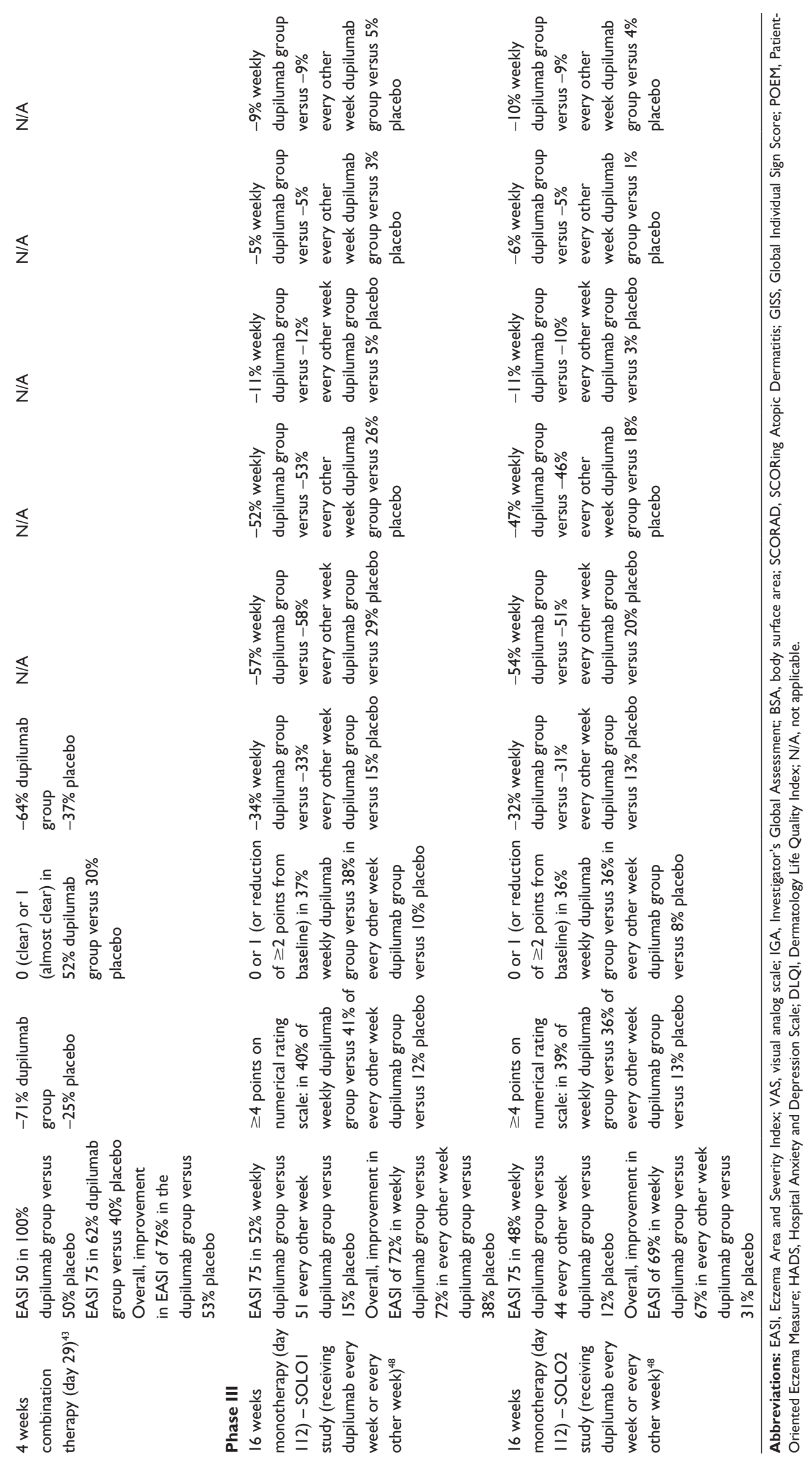


of AD (improvement in EASI, SCORAD, body surface area [BSA], pruritus [visual analog scale $\{\mathrm{VAS}\}$ ], Investigator's Global Assessment [IGA], Global Individual Sign Score [GISS]) as well as scale value of anxiety, depression, and quality of life, as compared with placebo (Table 1). ${ }^{48}$

The data published are promising, and trials of longer duration are needed to assess the long-term effectiveness and safety of dupilumab. ${ }^{48}$

\section{New treatment era for AD}

The approval for the marketization of dupilumab is approaching, and it may offer a significant therapeutic advancement in AD treatment. Unlike the currently available systemic treatments, dupilumab is going to profoundly change the therapeutic paradigm and the long-term management of AD, inaugurating a novel efficacious targeted therapeutic approach for patients with moderate-to-severe AD. Similar to biologic agents approved for psoriasis, dupilumab seems to offer the opportunity of a long-term control of the disease, being effective and overall safe. .33,36,37,40,43,48 $^{\text {. }}$

Dupilumab recently received US Food and Drug Administration breakthrough therapy designation for AD, with ongoing trials in both adult and pediatric populations. $^{33,40,43,48}$

Although the results of this study are thrilling, it is still not possible to translate the published results to the children, the most commonly affected population, since only patients aged $>18$ years were enrolled up to now. ${ }^{33,40,43,48,49}$ The future use of dupilumab in pediatric patients could potentially provide insights about its impact as early intervention in modifying the course of the disease.

Finally, pharmacoeconomically, we do not know how much this medication will cost; compared to the other biologics used in dermatology, it would be thought that dupilumab will likely cost tens to thousands of euro per year, which is significantly higher than the currently available systemic medications. ${ }^{33}$ As a consequence, dupilumab will likely be reserved for cases of severe AD unresponsive to traditional modalities. ${ }^{33}$ Currently, there are no studies that compare dupilumab to other medications, and it is difficult to compare the results from Phase I, II, and III studies involving dupilumab to the results achieved with other drugs. The tolerability of dupilumab seems high, maybe higher than that of systemic medications available; even the long-term side effects are not known.

\section{Conclusion}

With no doubts, dupilumab is ready to inaugurate a long and promising biological target treatment option for Th2 cell-mediated atopic immune response that characterizes AD and asthma. ${ }^{33,43,48}$ Interestingly, mepolizumab (antiIL-5) and omalizumab (anti-IgE), which have been studied in asthma with the same success as that of dupilumab, did not achieve enough results in AD. This fact may underlie a pivotal role of IL-4 and IL-13 among the Th-2 signal cytokines in AD.

Dupilumab initiates a decade that will be probably characterized by a great number of clinical trials in these areas, focusing not only on IL-4 and IL-13 but also on IL-31, IL-22, and TSLP, providing new hopes and insights for future therapeutic (and maybe prevention) approaches in $\mathrm{AD} .^{33,35,50}$

\section{Disclosure}

The authors declare no affiliation or significant financial involvement in any organizations or entity with a direct financial interest in the subject matter or materials discussed in the present manuscript. The authors report no conflicts of interest in this work.

\section{References}

1. Shaw TE, Currie GP, Koudelka CW, Simpson EL. Eczema prevalence in the United States: data from the 2003 National Survey of Children's Health. J Invest Dermatol. 2011;131(1):67-73.

2. Odhiambo JA, Williams HC, Clayton TO, Robertson CF, Asher MI; ISAAC Phase Three Study Group. Global variations in prevalence of eczema symptoms in children from ISAAC Phase Three. J Allergy Clin Immunol. 2009;124(6):1251-1258.e23.

3. Bos JD, Brenninkmeijer EE, Schram ME, Middelkamp-Hup MA, Spuls PI, Smitt JH. Atopic eczema or atopiform dermatitis. Exp Dermatol. 2010;19(4):325-331.

4. Bieber T. Atopic dermatitis. Ann Dermatol. 2010;22(2):125-137.

5. Malajian D, Guttman-Yassky E. New pathogenic and therapeutic paradigms in atopic dermatitis. Cytokine. 2015;73(2):311-318.

6. Leung DY, Guttman-Yassky E. Deciphering the complexities of atopic dermatitis: shifting paradigms in treatment approaches. $J$ Allergy Clin Immunol. 2014;134(4):769-779.

7. Romagnani S. The increased prevalence of allergy and the hygiene hypothesis: missing immune deviation, reduced immune suppression, or both? Immunology. 2004;112(3):352-363.

8. Garn H, Renz H. Epidemiological and immunological evidence for the hygiene hypothesis. Immunobiology. 2007;212(6):441-452.

9. Gittler JK, Krueger JG, Guttman-Yassky E. Atopic dermatitis results in intrinsic barrier and immune abnormalities: implications for contact dermatitis. J Allergy Clin Immunol. 2013;131(2):300-313.

10. Seidenari S, Giusti G. Objective assessment of the skin of children affected by atopic dermatitis: a study of $\mathrm{pH}$, capacitance and TEWL in eczematous and clinically uninvolved skin. Acta Derm Venereol. 1995; 75(6):429-433.

11. Guttman-Yassky E, Suárez-Fariñas M, Chiricozzi A, et al. Broad defects in epidermal cornification in atopic dermatitis identified through genomic analysis. J Allergy Clin Immunol. 2009;124(6):1235-1244.e58.

12. Guttman-Yassky E, Krueger JG. Psoriasis: evolution of pathogenic concepts and new therapies through phases of translational research. Br J Dermatol. 2007;157(6):1103-1115.

13. Suárez-Fariñas M, Tintle SJ, Shemer A, et al. Nonlesional atopic dermatitis skin is characterized by broad terminal differentiation defects and variable immune abnormalities. J Allergy Clin Immunol. 2011;127(4): 954-964.e1-e4. 
14. Boguniewicz M, Leung DY. Atopic dermatitis: a disease of altered skin barrier and immune dysregulation. Immunol Rev. 2011;242(1): 233-246.

15. Fujita H. The role of IL-22 and Th22 cells in human skin diseases. J Dermatol Sci. 2013;72(1):3-8.

16. Nograles KE, Zaba LC, Shemer A, et al. IL-22-producing “T22" $T$ cells account for upregulated IL-22 in atopic dermatitis despite reduced IL-17-producing TH17 T cells. J Allergy Clin Immunol. 2009;123(6): 1244-1252.e2.

17. Zheng Y, Danilenko DM, Valdez P, et al. Interleukin-22, a $T(H) 17$ cytokine, mediates IL-23-induced dermal inflammation and acanthosis. Nature. 2007;445(7128):648-651.

18. Kaminishi K, Soma Y, Kawa Y, Mizoguchi M. Flow cytometric analysis of IL-4, IL-13 and IFN-gamma expression in peripheral blood mononuclear cells and detection of circulating IL-13 in patients with atopic dermatitis provide evidence for the involvement of type 2 cytokines in the disease. J Dermatol Sci. 2002;29(1):19-25.

19. Akkoc T, de Koning PJ, Rückert B, Barlan I, Akdis M, Akdis CA Increased activation-induced cell death of high IFN-gammaproducing $\mathrm{T}(\mathrm{H}) 1$ cells as a mechanism of $\mathrm{T}(\mathrm{H}) 2$ predominance in atopic diseases. J Allergy Clin Immunol. 2008;121(3):652-658.e1.

20. Suárez-Fariñas M, Dhingra N, Gittler J, et al. Intrinsic atopic dermatitis shows similar TH2 and higher TH17 immune activation compared with extrinsic atopic dermatitis. J Allergy Clin Immunol. 2013;132(2): 361-370.

21. Flohr C, Mann J. New insights into the epidemiology of childhood atopic dermatitis. Allergy. 2014;69(1):3-16.

22. Gooderham M, Lynde CW, Papp K, et al. Review of systemic treatment options for adult atopic dermatitis. J Cutan Med Surg. 2017;21(1): 31-39.

23. Bieber T. Atopic dermatitis 2.0: from the clinical phenotype to the molecular taxonomy and stratified medicine. Allergy. 2012;67(12): 1475-1482.

24. D'Erme AM, Wilsmann-Theis D, Wagenpfeil J, et al. IL-36 (IL-1F9) is a biomarker for psoriasis skin lesions. J Invest Dermatol. 2015; 135(4):1025-1032.

25. Hello M, Aubert H, Bernier C, Néel A, Barbarot S. Atopic dermatitis of the adult. Rev Med Interne. 2016;37(2):91-99.

26. Bieber T, D'Erme AM, Akdis C, et al. Clinical phenotypes and endophenotypes of atopic dermatitis: Where are we and where should we go? J Alllergy Clin Immunol. 2017;139(4S):S58-S64.

27. Bieber T, Akdis C, Lauener R, et al. Global Allergy Forum and 3rd Davos Declaration 2015: atopic dermatitis/eczema: challenges and opportunities toward precision medicine. Allergy. 2016;71(5):588-592.

28. Mansouri Y, Guttman-Yassky E. Immune pathways in atopic dermatitis, and definition of biomarkers through broad and targeted therapeutics. J Clin Med. 2015;4(5):858-873.

29. Wollenberg A, Oranje A, Deleuran M, et al; European Task Force on Atopic Dermatitis/EADV Eczema Task Force. ETFAD/EADV eczema task force 2015 position paper on diagnosis and treatment of atopic dermatitis in adult and paediatric patients. J Eur Acad Dermatol Venereol. 2016;30(5):729-747.

30. Ring J, Alomar A, Bieber T, et al; European Dermatology Forum (EDF); European Academy of Dermatology and Venereology (EADV); European Federation of Allergy (EFA); European Task Force on Atopic Dermatitis (ETFAD); European Society of Pediatric Dermatology (ESPD); Global Allergy and Asthma European Network (GA2LEN). Guidelines for treatment of atopic eczema (atopic dermatitis) part I. J Eur Acad Dermatol Venereol. 2012;26(8):1045-1060.

31. Ring J, Alomar A, Bieber T, et al; European Dermatology Forum; European Academy of Dermatology and Venereology; European Task Force on Atopic Dermatitis; European Federation of Allergy; European Society of Pediatric Dermatology; Global Allergy and Asthma European Network. Guidelines for treatment of atopic eczema (atopic dermatitis) Part II. J Eur Acad Dermatol Venereol. 2012;26(9):1176-1193.
32. D'Erme AM, Hohl D. Use of emollient in atopic dermatitis prevention. Dermatol Ther. 2016;29(4):286-287.

33. McGregor S, Farhangian ME, Feldman SR. Dupilumab for the treatment of atopic dermatitis: a clinical trial review. Expert Opin Biol Ther. 2015;15(11):1657-1660.

34. Notaro ER, Sidbury R. Systemic agents for severe atopic dermatitis in children. Paediatr Drugs. 2015;17(6):449-457.

35. Biedermann T, Werfel T. Status quo and prospects for systemic therapy of atopic dermatitis. Biologics ante portas. Hautarzt. 2015;66(2): 108-113.

36. D'Erme AM. The beginning of biological treatment era in the atopic dermatitis management. Dermatol Ther. 2016;29(3):208-209.

37. Lauffer F, Ring J. Target-oriented therapy: emerging drugs for atopic dermatitis. Expert Opin Emerg Drugs. 2016;21(1):81-89.

38. Wang HH, Li YC, Huang YC. Efficacy of omalizumab in patients with atopic dermatitis: a systematic review and meta-analysis. J Allergy Clin Immunol. 2016;138(6):1719-1722.e1.

39. Oldhoff JM, Darsow U, Werfel T, et al. Anti-IL-5 recombinant humanized monoclonal antibody (mepolizumab) for the treatment of atopic dermatitis. Allergy. 2005;60(5):693-696.

40. Blakely K, Gooderham M, Papp K. Dupilumab, a monoclonal antibody for atopic dermatitis: a review of current literature. Skin Therapy Lett. 2016;21(2):1-5.

41. Kovalenko P, DiCioccio AT, Davis JD, et al. Exploratory population PK analysis of dupilumab, a fully human monoclonal antibody against IL-4R $\alpha$, in atopic dermatitis patients and normal volunteers. CPT Pharmacometrics Syst Pharmacol. 2016;5(11):617-624.

42. Hamilton JD, Suárez-Fariñas M, Dhingra N, et al. Dupilumab improves the molecular signature in skin of patients with moderate-to-severe atopic dermatitis. J Allergy Clin Immunol. 2014;134(6):1293-1300.

43. Beck LA, Thaçi D, Hamilton JD, et al. Dupilumab treatment in adults with moderate-to-severe atopic dermatitis. N Engl J Med. 2014;371(2): 130-139.

44. Thaçi D, Simpson EL, Beck LA, et al. Efficacy and safety of dupilumab in adults with moderate-to-severe atopic dermatitis inadequately controlled by topical treatments: a randomised, placebo-controlled, dose-ranging phase 2b trial. Lancet. 2016;387(10013):40-52.

45. Simpson EL, Gadkari A, Worm M, et al. Dupilumab therapy provides clinically meaningful improvement in patient-reported outcomes (PROs): a phase IIb, randomized, placebo-controlled, clinical trial in adult patients with moderate to severe atopic dermatitis (AD). J Am Acad Dermatol. 2016;75(3):506-515.

46. Wollenberg A, Bieber T. Proactive therapy of atopic dermatitis - an emerging concept. Allergy. 2009;64(2):276-278.

47. Hamilton JD, Ungar B, Guttman-Yassky E. Drug evaluation review: dupilumab in atopic dermatitis. Immunotherapy. 2015;7(10):1043-1058.

48. Simpson EL, Bieber T, Guttman-Yassky E, et al; SOLO 1 and SOLO 2 Investigators. Two phase 3 trials of dupilumab versus placebo in atopic dermatitis. N Engl J Med. 2016;375(24):2335-2348.

49. Santini G, Mores N, Malerba M, et al. Dupilumab for the treatment of asthma. Expert Opin Investig Drugs. 2017;26(3):357-366.

50. Ozdemir C. Monoclonal antibodies in allergy; updated applications and promising trials. Recent Pat Inflamm Allergy Drug Discov. 2015; 9(1):54-65. 


\section{Publish your work in this journal}

Drug Design, Development and Therapy is an international, peerreviewed open-access journal that spans the spectrum of drug design and development through to clinical applications. Clinical outcomes, patient safety, and programs for the development and effective, safe, and sustained use of medicines are the features of the journal, which

has also been accepted for indexing on PubMed Central. The manuscript management system is completely online and includes a very quick and fair peer-review system, which is all easy to use. Visit http://www.dovepress.com/testimonials.php to read real quotes from published authors.

Submit your manuscript here: http://www.dovepress.com/drug-design-development-and-therapy-journal 